\title{
Hybrid Chaos Synchronization of Hyperchaotic Lorenz and Hyperchaotic Chen Systems by Active Non-Linear Control
}

\author{
${ }^{1} \mathrm{~V}$. Sundarapandian and ${ }^{2} \mathrm{R}$. Karthikeyan \\ ${ }^{1}$ Systems and Control Engineering, Research and Development Centre, \\ Vel Tech Dr. RR \& Dr. SR Technical University, Avadi, 600-062 Chennai, Tamil Nadu, India \\ ${ }^{2}$ School of Electronics and Electrical Engineering, Singhania University, Pacheri Bari, \\ Dist. Jhunjhunu, 333-515 Rajastan, India
}

\begin{abstract}
In this study, researchers apply active non-linear control to achieve hybrid chaos synchronization of identical hyperchaotic Lorenz Systems in 2007, identical hyperchaotic Chen Systems in 2008 and non-identical hyperchaotic Lorenz and hyperchaotic Chen Systems. The hyperchaotic Lorenz System in 2007 and hyperchaotic Chen System in 2008 are important models of hyperchaotic systems. Hybrid synchronization of the hyperchaotic systems is achieved through the Complete Synchronization (CS) of the first and third states and Anti-synchronization (AS) of the second and fourth states. Since, the Lyapunov exponents are not required for these calculations, the Active Control Method is an effective and convenient method to achieve hybrid synchronization of the hyperchaotic systems. Numerical simulations are shown to demonstrate the effectiveness of the hybrid synchronization schemes derived in this study.
\end{abstract}

Key words: Active control, hybrid synchronization, hyperchaos, hyperchaotic Lorenz System, hyperchaotic Chen System, India

\section{INTRODUCTION}

Chaotic systems are non-linear dynamical systems that are highly sensitive to initial conditions. This sensitivity is popularly known as the butterfly effect (Alligood et al., 1997). Chaos synchronization has been widely studied in the last two decades (Alligood et al., 1997; Pecora and Carroll, 1990; Carroll and Pecora, 1991; Lakshmanan and Murali, 1996; Han et al., 1995; Blasius et al., 1999; Murali and Lakshmanan, 1998; Yang and Chua, 1999; Ott et al., 1990; Park and Kwon, 2003; Liao and Tsai, 2000; Yu and Zhang, 2006; Konishi et al., 1998; Agiza and Yassen, 2001; Huang et al., 2004; Ge and Chen, 2004; Zhang and Zhu, 2008; Qiang, 2007; Jian-Ping and Li, 2006; Li, 2008; Wang et al., 2007; Yan and Yu, 2008; Hahn, 1967).

Pecora and Carroll (1990) published a seminal paper on synchronization of two identical Chaotic Systems. From then on, chaos synchronization has been extensively explored in a variety of fields including physical systems (Carroll and Pecora, 1991), chemical systems (Lakshmanan and Murali, 1996), ecological systems (Han et al., 1995), secure communications, (Blasius et al., 1999; Murali and Lakshmanan, 1998), etc. In most of the synchronization approaches, the master-slave or drive-response formalism is used. If a particular Chaotic System is called the master or drive system and another Chaotic System is called the slave or response system then the idea of synchronization is to use the output of the master system to control the slave system so that, the output of the response system tracks the output of the master system asymptotically.

Since, the seminal research by Pecora and Carroll (1990), a variety of impressive approaches have been proposed for the synchronization of the Chaotic Systems such as the sampled-data feedback synchronization method (Yang and Chua, 1999), OGY Method (Ott et al., 1990), time-delay feedback method (Park and Kwon, 2003), adaptive design method (Liao and Tsai, 2000), backstepping design method ( $\mathrm{Yu}$ and Zhang, 2006), sliding mode control method (Konishi et al., 1998), active control method (Agiza and Yassen, 2001; Huang et al., 2004), etc.

So far many types of synchronizing phenomenon have been developed such as complete synchronization (Pecora and Carroll, 1990), phase synchronization (Ge and Chen, 2004), anti-synchronization (Zhang and Zhu, 2008), projective synchronization (Qiang, 2007), generalized projective synchronization (Jian-Ping and Li, 2006), etc. Complete Synchronization (CS) is characterized by the

Corresponding Author: R. Karthikeyan, Department of Electronics and Instrumentation Engineering, Vel Tech Dr. RR \& Dr. SR Technical University, Avadi, 600-062 Chennai, Tamil Nadu, India 
equality of state variables evolving in time while Anti-synchronization (AS) is characterized by the disappearance of the sum of relevant state variables evolving in time. Projective Synchronization (PS) is characterized by the fact the master and slave systems could be synchronized up to a scaling factor whereas in Generalized Projective Synchronization (GPS), the responses of the synchronized dynamical systems synchronize up to a constant scaling matrix $\alpha$. It is easy to see that the complete synchronization and anti-synchronization are special cases of the generalized projective synchronization where the scaling matrix $\alpha=\mathrm{I}$ and $\alpha=-\mathrm{I}$, respectively. In hybrid synchronization of chaotic systems ( $\mathrm{Li}, 2008)$, one part of the systems is completely synchronized and the other part is anti-synchronized so that, Complete Synchronization (CS) and anti-synchronization (CS) coexist in the systems. The coexistence of CS and AS is very useful in applications such as secure communication, chaotic encryption schemes, etc. Hyperchaotic system is usually defined as a chaotic system with at least two positive exponents implying that its dynamics are expanded in several different directions simultaneously.

Thus, the hyperchaotic systems have more complex dynamical behavior than chaotic systems and they can be used to improve the security of a chaotic communication system. In this study, researchers derive new results for the hybrid chaos synchronization of hyperchaotic Lorenz System (Wang et al., 2007) and hyperchaotic Chen System (Yan and Yu, 2008) by active non-linear control.

\section{HYBRID SYNCHRONIZATION OF IDENTICAL HYPERCHAOTIC LORENZ SYSTEMS}

Theoretical results: In this study, researchers apply the active control method to derive new results for the global hybrid synchronization of identical hyperchaotic Lorenz Systems (Wang et al., 2007). Thus, the master system is described by the hyperchaotic Lorenz dynamics:

$$
\begin{aligned}
& \dot{\mathrm{x}}_{1}=\mathrm{a}\left(\mathrm{x}_{2}-\mathrm{x}_{1}\right) \\
& \dot{\mathrm{x}}_{2}=\mathrm{bx}_{1}-\mathrm{x}_{2}-\mathrm{x}_{1} \mathrm{x}_{3}+\mathrm{x}_{4} \\
& \dot{\mathrm{x}}_{3}=\mathrm{x}_{1} \mathrm{x}_{2}-\mathrm{cx}_{3} \\
& \dot{\mathrm{x}}_{4}=-\mathrm{dx}_{1}
\end{aligned}
$$

Where, $\mathrm{x}_{1}-\mathrm{x}_{4}$ are the state variables and a-d are positive parameters of the system. The slave system is described by the controlled hyperchaotic Lorenz dynamics:

$$
\begin{aligned}
& \dot{\mathrm{y}}_{1}=\mathrm{a}\left(\mathrm{y}_{2}-\mathrm{y}_{1}\right)+\mathrm{u}_{1} \\
& \dot{\mathrm{y}}_{2}=\mathrm{by}_{1}-\mathrm{y}_{2}-\mathrm{y}_{1} \mathrm{y}_{3}+\mathrm{y}_{4}+\mathrm{u}_{2} \\
& \dot{\mathrm{y}}_{3}=\mathrm{y}_{1} \mathrm{y}_{2}-\mathrm{cy}_{3}+\mathrm{u}_{3} \\
& \dot{\mathrm{y}}_{4}=-\mathrm{dy}_{1}+\mathrm{u}_{4}
\end{aligned}
$$
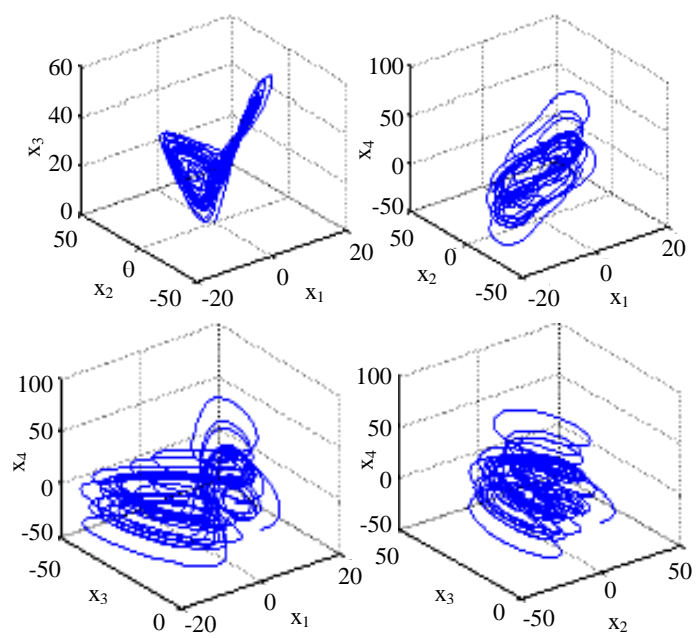

Fig. 1: State orbits of the hyperchaotic Lorenz System

Where, $\mathrm{y}_{1}-\mathrm{y}_{4}$ are the state variables and $\mathrm{u}_{1}-\mathrm{u}_{4}$ are the active controls to be designed. The hyperchaotic Lorenz System (Eq. 1) is a new hyperchaotic system derived from the Lorenz System by Wang et al. (2007). The system (Eq. 1) is hyperchaotic when:

$$
\mathrm{a}=10, \mathrm{~b}=28, \mathrm{c}=8 / 3 \text { and } \mathrm{d}=5
$$

The hyperchaotic portrait of the Lorenz System (Eq. 1) is shown in Fig. 1. For the hybrid synchronization of the hyperchaotic systems (Eq. 1 and 2), the errors are defined as:

$$
\begin{array}{ll}
\mathrm{e}_{1}=\mathrm{y}_{1}-\mathrm{x}_{1}, & \mathrm{e}_{2}=\mathrm{y}_{2}+\mathrm{x}_{2} \\
\mathrm{e}_{3}=\mathrm{y}_{3}-\mathrm{x}_{3}, & \mathrm{e}_{4}=\mathrm{y}_{4}+\mathrm{x}_{4}
\end{array}
$$

From the error Eq. 3, it is obvious that one part of the hyperchaotic systems is completely synchronized (first and third states) while the other part is anti-synchronized (second and last states) so that Complete Synchronization (CS) and Antisynchronization (AS) coexist in the synchronization process. A simple calculation gives the error dynamics as:

$$
\begin{aligned}
& \dot{\mathrm{e}}_{1}=\mathrm{a}\left(\mathrm{e}_{2}-\mathrm{e}_{1}\right)-2 \mathrm{ax}_{2}+\mathrm{u}_{1} \\
& \dot{\mathrm{e}}_{2}=\mathrm{be}_{1}-\mathrm{e}_{2}+\mathrm{e}_{4}+2 \mathrm{bx}_{1}-\mathrm{y}_{1} \mathrm{y}_{3}+\mathrm{x}_{1} \mathrm{x}_{3}+\mathrm{u}_{2} \\
& \dot{\mathrm{e}}_{3}=-\mathrm{ce}_{3}+\mathrm{y}_{1} \mathrm{y}_{2}-\mathrm{x}_{1} \mathrm{x}_{2}+\mathrm{u}_{3} \\
& \dot{\mathrm{e}}_{4}=-\mathrm{de}_{1}-2 \mathrm{dx}_{1}+\mathrm{u}_{4}
\end{aligned}
$$

Researchers consider the non-linear controller defined by: 


$$
\begin{aligned}
& \mathrm{u}_{1}=-\mathrm{ae}_{2}+2 \mathrm{ax}_{2} \\
& \mathrm{u}_{2}=-\mathrm{be}_{1}-\mathrm{e}_{4}-2 \mathrm{bx}_{1}+\mathrm{y}_{1} \mathrm{y}_{3}+\mathrm{x}_{1} \mathrm{x}_{3} \\
& \mathrm{u}_{3}=-\mathrm{y}_{1} \mathrm{y}_{2}+\mathrm{x}_{1} \mathrm{x}_{2} \\
& \mathrm{u}_{4}=-\mathrm{e}_{4}+\mathrm{de}_{1}+2 \mathrm{dx}_{1}
\end{aligned}
$$

Substituting Eq. 5 into 4, researchers get the error dynamics as:

$$
\begin{array}{ll}
\dot{\mathrm{e}}_{1}=-\mathrm{ae}_{1}, & \dot{\mathrm{e}}_{2}=-\mathrm{e}_{2} \\
\dot{\mathrm{e}}_{3}=-\mathrm{ce}_{3}, & \dot{\mathrm{e}}_{4}=-\mathrm{e}_{4}
\end{array}
$$

The candidate Lyapunov function is taken as:

$$
\mathrm{V}(\mathrm{e})=\frac{1}{2} \mathrm{e}^{\mathrm{T}} \mathrm{e}=\frac{1}{2}\left(\mathrm{e}_{1}^{2}+\mathrm{e}_{2}^{2}+\mathrm{e}_{3}^{2}+\mathrm{e}_{4}^{2}\right)
$$

which is a positive definite function on $\mathrm{R}^{4}$. Differentiating Eq. 7 along the trajectories of Eq. 6, we get:

$$
\dot{\mathrm{V}}(\mathrm{e})=-\mathrm{ae}_{1}^{2}-\mathrm{e}_{2}^{2}-\mathrm{ce}_{3}^{2}-\mathrm{e}_{4}^{2}
$$

which is a negative definite function on $\mathrm{R}^{4}$. Thus by Lyapunov Stability Theory (Hahn, 1967), the error dynamics Eq. 6 is globally exponentially stable. Hence, we obtain the following result.

Theorem 1: The identical hyperchaotic Lorenz Systems (Eq. 1 and 2) are globally and exponentially hybrid synchronized by the active non-linear controller (Eq. 5).

Numerical results: For the numerical simulations, the 4th-order Runge-Kutta Method with time-step $\mathrm{h}=10^{-6}$ is used to solve the two systems of differential Eq. 1 and 2 with the active non-linear controller (Eq. 5). The parameters of the hyperchaotic Lorenz Systems (Eq. 1 and 2) are chosen so that, the systems are hyperchaotic, i.e.:

$$
\mathrm{a}=10, \mathrm{~b}=28, \mathrm{c}=8 / 3 \text { and } \mathrm{d}=5
$$

The initial values of the master system (Eq. 1) are chosen as:

$$
\mathrm{x}_{1}(0)=18, \mathrm{x}_{2}(0)=12, \mathrm{x}_{3}(0)=8, \mathrm{x}_{4}(0)=15
$$

The initial values of the slave system (Eq. 2) are chosen as:

$$
\mathrm{y}_{1}(0)=26, \mathrm{y}_{2}(0)=22, \mathrm{y}_{3}(0)=18, \mathrm{y}_{4}(0)=4
$$

Figure 2 shows the hybrid synchronization of the hyperchaotic Lorenz Systems (Eq. 1 and 2):
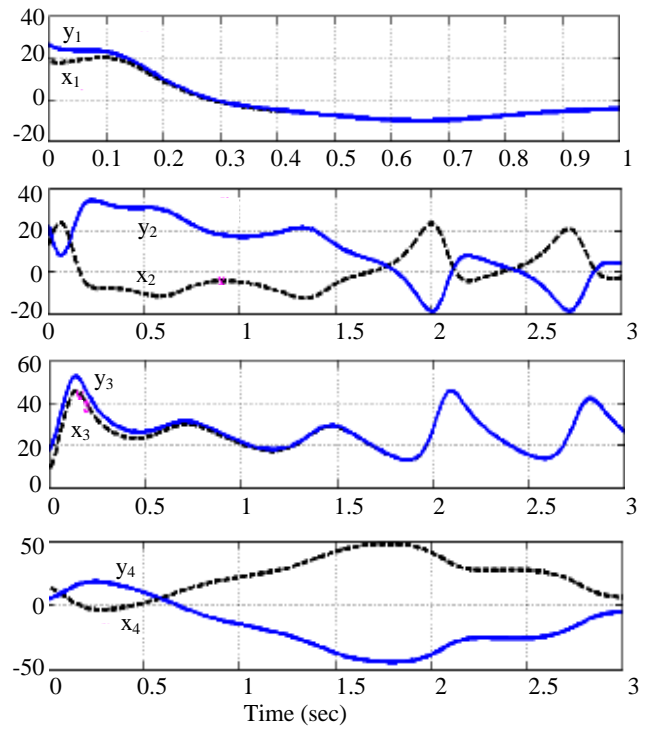

Fig. 2: Hybrid synchronization of hyperchaotic Lorenz Systems

\section{HYBRID SYNCHRONIZATION OF IDENTICAL HYPERCHAOTIC CHEN SYSTEMS}

Theoretical results: In this study, researchers apply the active control method to derive new results for the global hybrid synchronization of identical hyperchaotic Chen Systems (Yan and $\mathrm{Yu}, 2008$ ). Thus, the master system is described by the hyperchaotic Chen dynamics:

$$
\begin{aligned}
& \dot{\mathrm{x}}_{1}=\alpha\left(\mathrm{x}_{2}-\mathrm{x}_{1}\right) \\
& \dot{\mathrm{x}}_{2}=(\gamma-\alpha) \mathrm{x}_{1}+\gamma \mathrm{x}_{2}-\mathrm{x}_{1} \mathrm{x}_{3} \\
& \dot{\mathrm{x}}_{3}=-\beta \mathrm{x}_{3}-\mathrm{x}_{4}+\mathrm{x}_{1} \mathrm{x}_{2}-\mathrm{x}_{2} \mathrm{x}_{3}+\mathrm{x}_{1} \mathrm{x}_{3} \\
& \dot{\mathrm{x}}_{4}=-\delta \mathrm{x}_{4}+\mathrm{x}_{2} \mathrm{x}_{3}-\mathrm{x}_{1} \mathrm{x}_{3}
\end{aligned}
$$

Where, $\mathrm{x}_{1}-\mathrm{x}_{4}$ are the state variables and $\alpha, \beta, \gamma$ and $\delta$ are positive parameters of the system. The slave system is described by the controlled hyperchaotic Chen dynamics:

$$
\begin{aligned}
& \dot{\mathrm{y}}_{1}=\alpha\left(\mathrm{y}_{2}-\mathrm{y}_{1}\right)+\mathrm{u}_{1} \\
& \dot{\mathrm{y}}_{2}=(\gamma-\alpha) \mathrm{y}_{1}+\gamma \mathrm{y}_{2}-\mathrm{y}_{1} \mathrm{y}_{3}+\mathrm{u}_{2} \\
& \dot{\mathrm{y}}_{3}=-\beta \mathrm{y}_{3}-\mathrm{y}_{4}+\mathrm{y}_{1} \mathrm{y}_{2}-\mathrm{y}_{2} \mathrm{y}_{3}+\mathrm{y}_{1} \mathrm{y}_{3}+\mathrm{u}_{3} \\
& \dot{\mathrm{y}}_{4}=-\delta \mathrm{y}_{4}+\mathrm{y}_{2} \mathrm{y}_{3}-\mathrm{y}_{1} \mathrm{y}_{3}+\mathrm{u}_{4}
\end{aligned}
$$

Where, $\mathrm{y}_{1}-\mathrm{y}_{4}$ are the state variables and $\mathrm{u}_{1}-\mathrm{u}_{4}$ are the active controls to be designed. The hyperchaotic Chen System (Eq. 9) is a new hyperchaotic system derived from the Chen System by Yan and Yu (2008). The system (Eq. 9) is hyperchaotic when:

$$
\alpha=37, \beta=3, \gamma=26 \text { and } \delta=38
$$



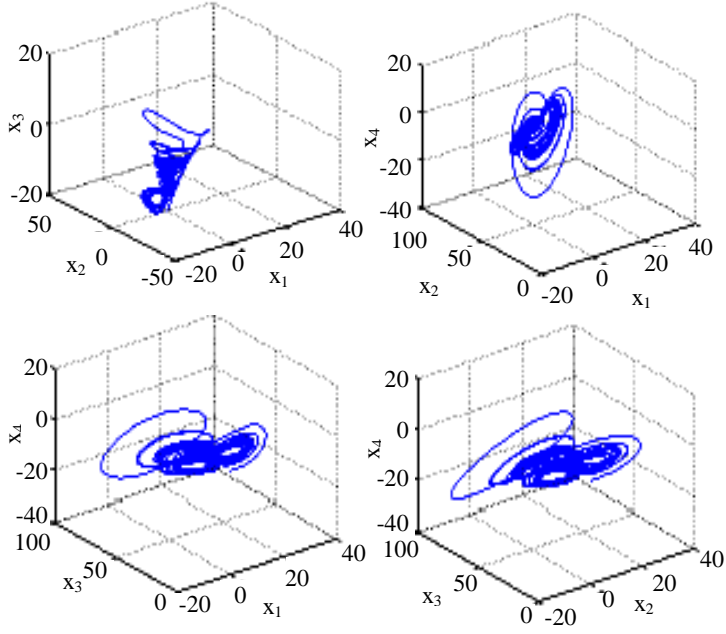

Fig. 3: State orbits of the hyperchaotic Chen System

The state orbits of the hyperchaotic Chen System (Eq. 9) are shown in Fig. 3. For the hybrid synchronization of the hyperchaotic systems (Eq. 9 and 10), the errors are defined as:

$$
\begin{array}{ll}
\mathrm{e}_{1}=\mathrm{y}_{1}-\mathrm{x}_{1}, & \mathrm{e}_{2}=\mathrm{y}_{2}+\mathrm{x}_{2} \\
\mathrm{e}_{3}=\mathrm{y}_{3}-\mathrm{x}_{3}, & \mathrm{e}_{4}=\mathrm{y}_{4}+\mathrm{x}_{4}
\end{array}
$$

From the error Eq. 11, it is obvious that one part of the hyperchaotic systems is completely synchronized (first and third states) while the other part is antisynchronized (second and last states) so that, Complete Synchronization (CS) and Anti-synchronization (AS) coexist in the synchronization process. A simple calculation gives the error dynamics as:

$$
\begin{aligned}
\dot{\mathrm{e}}_{1}= & \alpha\left(\mathrm{e}_{2}-\mathrm{e}_{1}\right)-2 \alpha \mathrm{x}_{2}+\mathrm{u}_{1} \\
\dot{\mathrm{e}}_{2}= & (\gamma-\alpha) \mathrm{e}_{1}+\gamma \mathrm{e}_{2}+2(\gamma-\alpha) \mathrm{x}_{1}-\mathrm{y}_{1} \mathrm{y}_{3}-\mathrm{x}_{1} \mathrm{x}_{3}+\mathrm{u}_{2} \\
\dot{\mathrm{e}}_{3}= & -\beta \mathrm{e}_{3}-\mathrm{e}_{4}+2 \mathrm{x}_{4}+\mathrm{y}_{1} \mathrm{y}_{2}-\mathrm{y}_{2} \mathrm{y}_{3}+\mathrm{y}_{1} \mathrm{y}_{3}- \\
& \mathrm{x}_{1} \mathrm{x}_{2}+\mathrm{x}_{2} \mathrm{x}_{3}-\mathrm{x}_{1} \mathrm{x}_{3}+\mathrm{u}_{3} \\
\dot{\mathrm{e}}_{4}= & -\delta \mathrm{e}_{4}+\mathrm{y}_{2} \mathrm{y}_{3}-\mathrm{y}_{1} \mathrm{y}_{3}+\mathrm{x}_{2} \mathrm{x}_{3}-\mathrm{x}_{1} \mathrm{x}_{3}+\mathrm{u}_{4}
\end{aligned}
$$

Researchers consider the non-linear controller defined by:

$$
\begin{aligned}
\mathrm{u}_{1}= & -\alpha \mathrm{e}_{2}+2 \alpha \mathrm{x}_{2} \\
\mathrm{u}_{2}= & -(\gamma-\alpha) \mathrm{e}_{1}-(\gamma+1) \mathrm{e}_{2}-2(\gamma-\alpha) \mathrm{x}_{1}+\mathrm{y}_{1} \mathrm{y}_{3}+\mathrm{x}_{1} \mathrm{x}_{3} \\
\mathrm{u}_{3}= & \mathrm{e}_{4}-2 \mathrm{x}_{4}-\mathrm{y}_{1} \mathrm{y}_{2}+\mathrm{y}_{2} \mathrm{y}_{3}-\mathrm{y}_{1} \mathrm{y}_{3}+ \\
& \mathrm{x}_{1} \mathrm{x}_{2}-\mathrm{x}_{2} \mathrm{x}_{3}+\mathrm{x}_{1} \mathrm{x}_{3} \\
\mathrm{u}_{4}= & -\mathrm{y}_{2} \mathrm{y}_{3}+\mathrm{y}_{1} \mathrm{y}_{3}-\mathrm{x}_{2} \mathrm{x}_{3}+\mathrm{x}_{1} \mathrm{x}_{3}
\end{aligned}
$$

Substituting Eq. 13 into 12, researchers get the error dynamics as:

$$
\begin{array}{ll}
\dot{\mathrm{e}}_{1}=-\alpha \mathrm{e}_{1}, & \dot{\mathrm{e}}_{2}=-\mathrm{e}_{2} \\
\dot{\mathrm{e}}_{3}=-\beta \mathrm{e}_{3}, & \dot{\mathrm{e}}_{4}=-\delta \mathrm{e}_{4}
\end{array}
$$

The candidate Lyapunov function is taken as:

$$
\mathrm{V}(\mathrm{e})=\frac{1}{2} \mathrm{e}^{\mathrm{T}} \mathrm{e}=\frac{1}{2}\left(\mathrm{e}_{1}^{2}+\mathrm{e}_{2}^{2}+\mathrm{e}_{3}^{2}+\mathrm{e}_{4}^{2}\right)
$$

which is a positive definite function on $\mathrm{R}^{4}$. Differentiating Eq. 15 along the trajectories of Eq. 14, we get:

$$
\dot{\mathrm{V}}(\mathrm{e})=-\alpha \mathrm{e}_{1}^{2}-\mathrm{e}_{2}^{2}-\beta \mathrm{e}_{3}^{2}-\delta \mathrm{e}_{4}^{2}
$$

which is a negative definite function on $\mathrm{R}^{4}$. Thus by Lyapunov Stability Theory (Hahn, 1967), the error dynamics (Eq. 16) is globally exponentially stable. Hence, we obtain the following result for the hybrid synchronization of the identical hyperchaotic Chen Systems given by Eq. 9 and 10 .

Theorem 2: The identical hyperchaotic Chen Systems (Eq. 9 and 10) are globally and exponentially hybrid synchronized by the active non-linear controller (Eq. 13).

Numerical results: For the numerical simulations, the 4th-order Runge-Kutta Method with time-step $h=10^{-6}$ is used to solve the two systems of differential Eq. 9 and 10 with the active non-linear controller (Eq. 13). The parameters of the hyperchaotic Chen Systems (Eq. 9 and 10 ) are chosen so that the systems are hyperchaotic, i.e.:

$$
\alpha=37, \beta=3, \gamma=26 \text { and } \delta=38
$$

The initial values of the master system (Eq. 9) are chosen as:

$$
\mathrm{x}_{1}(0)=22, \mathrm{x}_{2}(0)=6, \mathrm{x}_{3}(0)=12, \mathrm{x}_{4}(0)=9
$$

The initial values of the slave system (Eq. 10) are chosen as:

$$
\mathrm{y}_{1}(0)=12, \mathrm{y}_{2}(0)=18, \mathrm{y}_{3}(0)=20, \mathrm{y}_{4}(0)=16
$$

Figure 4 shows the hybrid synchronization of the hyperchaotic Chen Systems (Eq. 9 and 10). We note that the odd states of the master and slave systems are completely synchronized while the even states of the master and slave systems are anti-synchronized. 

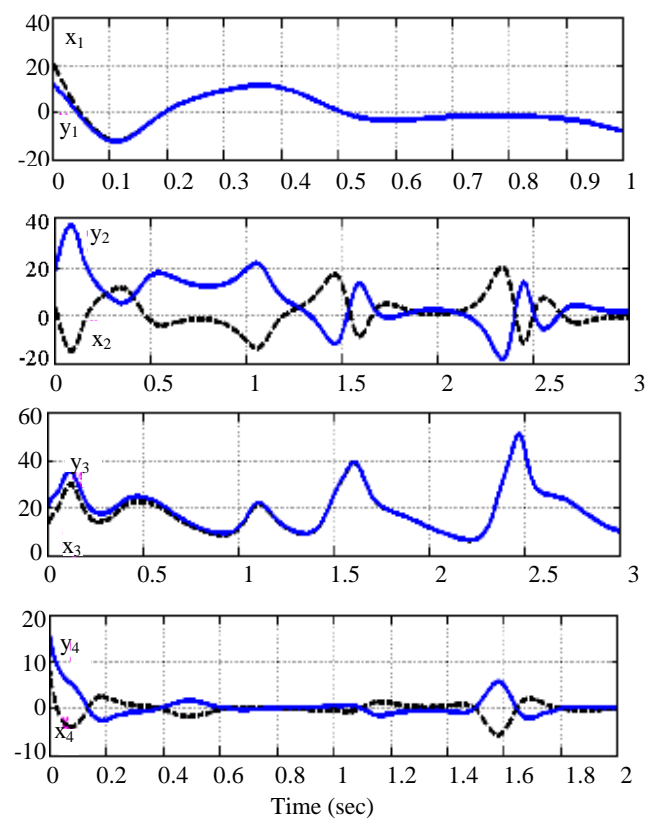

Fig. 4: Hybrid synchronization of hyperchaotic Chen Systems

\section{SYNCHRONIZATION OF NON-DENTICAL HYPERCHAOTIC LORENZ AND HYPERCHAOTIC CHEN SYSTEMS}

Theoretical results: In this study, researchers apply the Active Control Method to derive new results for the global hybrid synchronization of hyperchaotic Lorenz System in 2007 and hyperchaotic Chen System, 2008. As the master system, we consider the hyperchaotic Lorenz dynamics which is given by the equations:

$$
\begin{aligned}
& \dot{\mathrm{x}}_{1}=\mathrm{a}\left(\mathrm{x}_{2}-\mathrm{x}_{1}\right), \dot{\mathrm{x}}_{2}=\mathrm{bx}_{1}-\mathrm{x}_{2}-\mathrm{x}_{1} \mathrm{x}_{3}+\mathrm{x}_{4} \\
& \dot{\mathrm{x}}_{3}=\mathrm{x}_{1} \mathrm{x}_{2}-\mathrm{cx}_{3}, \dot{\mathrm{x}}_{4}=-\mathrm{dx}_{1}
\end{aligned}
$$

Where, $\mathrm{x}_{1}-\mathrm{x}_{4}$ are the state variables and a-d are positive parameters of the system. As the slave system, we consider the hyperchaotic Chen dynamics which is given by the equations:

$$
\begin{aligned}
& \dot{\mathrm{y}}_{1}=\alpha\left(\mathrm{y}_{2}-\mathrm{y}_{1}\right)+\mathrm{u}_{1} \\
& \dot{\mathrm{y}}_{2}=(\gamma-\alpha) \mathrm{y}_{1}+\gamma \mathrm{y}_{2}-\mathrm{y}_{1} \mathrm{y}_{3}+\mathrm{u}_{2} \\
& \dot{\mathrm{y}}_{3}=-\beta \mathrm{y}_{3}-\mathrm{y}_{4}+\mathrm{y}_{1} \mathrm{y}_{2}-\mathrm{y}_{2} \mathrm{y}_{3}+\mathrm{y}_{1} \mathrm{y}_{3}+\mathrm{u}_{3} \\
& \dot{\mathrm{y}}_{4}=-\delta \mathrm{y}_{4}+\mathrm{y}_{2} \mathrm{y}_{3}-\mathrm{y}_{1} \mathrm{y}_{3}+\mathrm{u}_{4}
\end{aligned}
$$

Where, $\mathrm{y}_{1}-\mathrm{y}_{4}$ are the state variables, $\alpha, \beta, \gamma$ and $\delta$ are parameters of the system and $\mathrm{u}_{1}-\mathrm{u}_{4}$ are the active controls to be designed. For the hybrid synchronization of the hyperchaotic systems (Eq. 17 and 18), the errors are defined as:

$$
\begin{array}{ll}
e_{1}=y_{1}-x_{1}, & e_{2}=y_{2}+x_{2} \\
e_{3}=y_{3}-x_{3}, & e_{4}=y_{4}+x_{4}
\end{array}
$$

From the error Eq. 19, it is obvious that one part of the hyperchaotic systems is completely synchronized (first and third states) while the other part is anti-synchronized (second and last states) so that, Complete Synchronization (CS) and Anti-synchronization (AS) co-exist in the synchronization process. A simple calculation gives the error dynamics as:

$$
\begin{aligned}
\dot{\mathrm{e}}_{1}=\alpha\left(\mathrm{e}_{2}-\mathrm{e}_{1}\right)+(\mathrm{a}-\alpha) \mathrm{x}_{1}-(\mathrm{a}+\alpha) \mathrm{x}_{2}+\mathrm{u}_{1} \\
\dot{\mathrm{e}}_{2}=(\gamma-\alpha) \mathrm{e}_{1}+\gamma \mathrm{e}_{2}+(\gamma-\alpha+\mathrm{b}) \mathrm{x}_{1}- \\
\quad(\gamma+1) \mathrm{x}_{2}+\mathrm{x}_{4}-\mathrm{y}_{1} \mathrm{y}_{3}-\mathrm{x}_{1} \mathrm{x}_{3}+\mathrm{u}_{2} \\
\dot{\mathrm{e}}_{3}=-\beta \mathrm{e}_{3}-\mathrm{e}_{4}+(\mathrm{c}-\beta) \mathrm{x}_{3}+\mathrm{x}_{4}+\mathrm{y}_{1} \mathrm{y}_{2}- \\
\mathrm{y}_{2} \mathrm{y}_{3}+\mathrm{y}_{1} \mathrm{y}_{3}-\mathrm{x}_{1} \mathrm{x}_{2}+\mathrm{u}_{3} \\
\dot{\mathrm{e}}_{4}=-\delta \mathrm{e}_{4}+\delta \mathrm{x}_{4}-d \mathrm{x}_{1}+\mathrm{y}_{2} \mathrm{y}_{3}-\mathrm{y}_{1} \mathrm{y}_{3}+\mathrm{u}_{4}
\end{aligned}
$$

Researchers consider the non-linear controller defined by:

$$
\begin{aligned}
\mathrm{u}_{1}= & -\alpha \mathrm{e}_{2}-(\mathrm{a}-\alpha) \mathrm{x}_{1}+(\mathrm{a}+\alpha) \mathrm{x}_{2} \\
\mathrm{u}_{2}= & -(\gamma-\alpha) \mathrm{e}_{1}-(\gamma+1) \mathrm{e}_{2}-(\gamma-\alpha+b) \mathrm{x}_{1}+ \\
& (\gamma+1) \mathrm{x}_{2}-\mathrm{x}_{4}+\mathrm{y}_{1} \mathrm{y}_{3}+\mathrm{x}_{1} \mathrm{x}_{3} \\
\mathrm{u}_{3}= & \mathrm{e}_{4}-(\mathrm{c}-\beta) \mathrm{x}_{3}-\mathrm{x}_{4}-\mathrm{y}_{1} \mathrm{y}_{2}+\mathrm{y}_{2} \mathrm{y}_{3}-\mathrm{y}_{1} \mathrm{y}_{3}+\mathrm{x}_{1} \mathrm{x}_{2} \\
\mathrm{u}_{4}= & -\delta \mathrm{x}_{4}+d \mathrm{x}_{1}-\mathrm{y}_{2} \mathrm{y}_{3}+\mathrm{y}_{1} \mathrm{y}_{3}
\end{aligned}
$$

Substituting Eq. 21 into 20, we get the error dynamics as:

$$
\begin{array}{ll}
\dot{\mathrm{e}}_{1}=-\alpha \mathrm{e}_{1}, & \dot{\mathrm{e}}_{2}=-\mathrm{e}_{2} \\
\dot{\mathrm{e}}_{3}=-\beta \mathrm{e}_{3}, & \dot{\mathrm{e}}_{4}=-\delta \mathrm{e}_{4}
\end{array}
$$

The candidate Lyapunov function is taken as:

$$
\mathrm{V}(\mathrm{e})=\frac{1}{2} \mathrm{e}^{\mathrm{T}} \mathrm{e}=\frac{1}{2}\left(\mathrm{e}_{1}^{2}+\mathrm{e}_{2}^{2}+\mathrm{e}_{3}^{2}+\mathrm{e}_{4}^{2}\right)
$$

which is a positive definite function on $\mathrm{R}^{4}$. Differentiating Eq. 23 along the trajectories of Eq. 22, we get:

$$
\dot{\mathrm{V}}(\mathrm{e})=-\alpha \mathrm{e}_{1}^{2}-\mathrm{e}_{2}^{2}-\beta \mathrm{e}_{3}^{2}+\delta \mathrm{e}_{4}^{2}
$$

which is a negative definite function on $\mathrm{R}^{4}$. Thus by Lyapunov Stability Theory (Hahn, 1967), the error 
dynamics (Eq. 22) is globally exponentially stable. Hence, we obtain the following result.

Theorem 3: The non-identical hyperchaotic Lorenz system (Eq. 17) and hyperchaotic Chen System (Eq. 18) are globally and exponentially hybrid synchronized by the active non-linear controller (Eq. 21).

Numerical results: For the numerical simulations, the 4th-order Runge-Kutta Method with time-step $\mathrm{h}=10^{-6}$ is used to solve the two systems of differential Eq. 17 and 18 with the active non-linear controller (Eq. 21). The parameters of the hyperchaotic Lorenz System (Eq. 17) are chosen as:

$$
\mathrm{a}=10, \mathrm{~b}=28, \mathrm{c}=8 / 3 \text { and } \mathrm{d}=5
$$

The parameters of the hyperchaotic Chen System (Eq. 18) are chosen as:

$$
\alpha=37, \beta=3, \gamma=26, \delta=38
$$

The initial values of the master system (Eq. 17) are chosen as:

$$
x_{1}(0)=16, x_{2}(0)=22, x_{3}(0)=9, x_{4}(0)=25
$$

The initial values of the slave system (Eq. 18) are chosen as:

$$
\mathrm{y}_{1}(0)=6, \mathrm{y}_{2}(0)=8, \mathrm{y}_{3}(0)=24, \mathrm{y}_{4}(0)=12
$$

Figure 5 shows the hybrid synchronization between the hyperchaotic Lorenz and hyperchaotic Chen Systems.
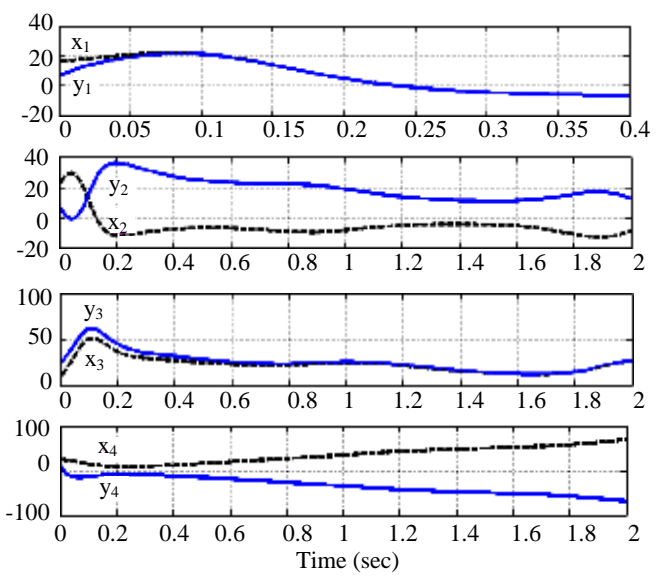

Fig. 5: Hybrid Synchronization of hyperchaotic Lorenz and hyperchaotic Chen Systems

\section{CONCLUSION}

In this study, researchers deployed active Non-linear Control Method for the global hybrid synchronization of identical hyperchaotic Lorenz Systems in 2007, identical hyperchaotic Chen Systems in 2008 and non-identical hyperchaotic Lorenz System in 2007 and hyperchaotic Chen System in 2008. Since, Lyapunov exponents are not required for these calculations, the proposed active nonlinear control method is effective and convenient to achieve the global hybrid synchronization of the identical and non-identical hyperchaotic Lorenz and hyperchaotic Chen Systems. Numerical simulations are shown to illustrate the effectiveness of the hybrid synchronization schemes derived in this study.

\section{REFERENCES}

Agiza, H.N. and M.T. Yassen, 2001. Synchronization of rossler and chen chaotic dynamical systems using active control. Phys. Lett. A, 278: 191-197.

Alligood, K.T., T. Sauer and J.A. Yorke, 1997. Chaos: An Introduction to Dynamical Systems, Springer-Verlag, New York.

Blasius, B., A. Huppert and L. Stone, 1999. Complex dynamics and phase synchronization in spatially extended ecological system. Nature, 399: 354-359.

Carroll, T.L. and L.M. Pecora, 1991. Synchronizing chaotic circuits. IEEE Trans. Circuits Syst., 38: 453-456.

Ge, Z.M. and C.C. Chen, 2004. Phase synchronization of coupled chaotic multiple time scales systems. Chaos Solitons Fractals, 20: 639-647.

Hahn, W., 1967. The Stability of Motion. Springer-Verlag, Berlin.

Han, S.K., C. Kerrer and Y. Kuramoto, 1995. Dephasing and bursting in coupled neural oscillators. Phys. Rev. Lett., 75: 3190-3193.

Huang, L., R. Feng and M. Wang, 2004. Synchronization of chaotic systems via nonlinear control. Phys. Lett. A, 320: 271-275.

Jian-Ping, Y. and C.P. Li, 2006. Generalized projective synchronization for the chaotic lorenz system and the chaotic chen system. J. Shanghai Univ., 10: 299-304.

Konishi, K., M. Hirai and H. Kokame, 1998. Sliding mode control for a class of chaotic systems. Phys. Lett. A, 245: 511-517.

Lakshmanan, M. and K. Murali, 1996. Chaos in Nonlinear Oscillators: Controlling and Synchronization. World Scientific, Singapore.

Li, R.H., 2008. A special full-state hybrid synchronization in symmetrical chaotic systems. Applied Math. Comput., 200: 321-329. 
Liao, T.L. and S.H. Tsai, 2000. Adaptive synchronization of chaotic systems and its application to secure communications. Chaos Solitons Fractals, 11: 1387-1396.

Murali, K. and M. Lakshmanan, 1998. Secure communication using a compound signal from generalized synchronizable chaotic systems. Phys. Lett. A, 241: 303-310.

Ott, E., C. Grebogi and J.A. Yorke, 1990. Controlling chaos. Phys. Rev. Lett., 64: 1196-1199.

Park, J.H. and O.M. Kwon, 2003. A novel criterion for delayed feedback control of time-delay chaotic systems. Chaos Solitons Fractals, 17: 709-716.

Pecora, L.M. and T.L. Carroll, 1990. Synchronization in chaotic systems. Phys. Rev. Lett., 64: 821-824.

Qiang, J., 2007. Projective synchronization of a new hyperchaotic lorenz system. Phys. Lett. A, 370: 40-45.
Wang, G.Y., Y. Zheng and J. Liu, 2007. A hyperchaotic lorenz attractor and its circuit implementation. Acta Phys. Sin., 56: 3113-3120.

Yan, Z. and P. Yu, 2008. Hyperchaos synchronization and control on a new hyperchaotic attractor. Chaos Solitons Fractals, 35: 333-345.

Yang, T. and L.O. Chua, 1999. Control of chaos using sampled-data feedback control. Int. J. Bifurcation Chaos, 9: 215-219.

$\mathrm{Yu}$, Y.G. and S.C. Zhang, 2006. Adaptive backstepping synchronization of uncertain chaotic systems. Chaos Solitons Fractals, 27: 1369-1375.

Zhang, X. and H. Zhu, 2008. Anti-synchronization of two different hyperchaotic systems via active and adaptive control. Int. J. Nonlinear Sci., 6: 216-223. 